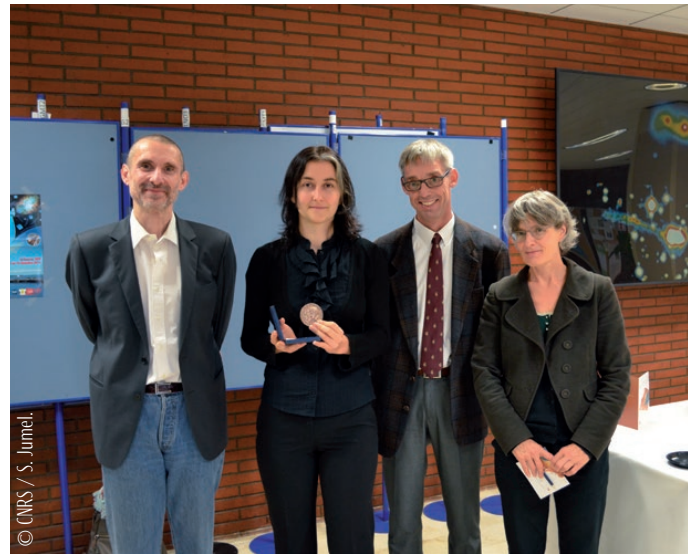

\title{
Lenka Zdeborová, une mathémaphysinformaticienne en bronze
}

Spécialiste de physique statistique, cette théoricienne se passionne pour les systèmes désordonnés, l'informatique théorique ou encore l'algorithmique. Elle fait partie des lauréats 2014 de la médaille de bronze du CNRS.

Remise de la médaille de bronze du CNRS à Lenka Zdeborová, le 24 octobre 2014

à I'IPhT (Saclay). De gauche à droite : Michel Bauer, Directeur de I'IPhT, Lenka Zdeborová, Bart van Tiggelen, Directeur adjoint scientifique de l'INP,

et Véronique Debisschop, Déléguée régionale CNRS Ile-de-France Sud.
"Aux mathématiciens je dis que je suis physicienne, et aux physiciens que je fais des mathématiques appliquées à l'informatique ", plaisante Lenka Zdeborová, chargée de recherche du CNRS à l'Institut de physique théorique (IPhT, CNRS/CEA) à Saclay. Une chose est sûre : tout juste médaillée de bronze du CNRS [1], cette scientifique d'origine tchèque possède à la fois la bosse des maths et la fibre physicienne. Spécialiste de physique statistique, elle applique son art à la résolution de complexes problèmes d'algorithmique. Et ne conçoit son activité que dans l'échange et le partage.

Pour la théoricienne, maths et physique ont toujours été une aventure collective : "Lycéenne, j'ai participé à de nombreuses olympiades scientifiques qui ont été pour moi l'occasion de rencontres importantes. " Et ont constitué un entraînement de choc pour la future chercheuse. À l'Institut Charles de Prague, la meilleure université scientifique de son pays d'origine, Lenka Zdeborová se prend de passion pour la physique statistique et les probabilités. D'où une thèse de master sur les verres de spins, des systèmes magnétiques désordonnés présentant une organisation microscopique d'une rare complexité.

De cette époque, la jeune femme garde surtout le souvenir du réseau scientifique européen auquel appartient alors son laboratoire. "Cela m'a permis de découvrir une communauté au-delà des frontières de la République tchèque. " Et par conséquent d'opter pour un doctorat en France. Spécialité : l'application des outils développés pour les systèmes physiques désordonnés à l'informatique, l'algorithmique et l'optimisation.

Elle explique : "Un exemple classique est celui du coloriage d'une carte : combien faut-il de couleurs pour garantir que deux territoires adjacents soient d'une couleur différente? Dans le cas général, la réponse est ardue. Or, on sait désormais que les outils de la physique statistique permettent un éclairage renouvelé. " Abscons pour le profane ? Sans aucun doute, comme en témoigne le titre de la thèse de la scientifique : Physique statistique des problèmes d'optimisation difficile!

Mais également riche d'applications. Ainsi, la théoricienne a développé une méthode qui permet d'identifier des communautés, c'est-à-dire des groupes très interconnectés sur un graphe, une tâche importante, par exemple, dans l'étude des réseaux sociaux. Elle détaille : "Pour ce faire, on considère un marcheur qui se déplace aléatoirement sur le graphe, mais sans jamais revenir sur ses pas. Par rapport aux autres méthodes pour ce problème, la nôtre a besoin de considérablement moins d'informations sur les connexions pour réussir. Ce travail a été poursuivi par de nombreux mathématiciens par la suite."

Un travail dont la qualité la conduit en 2008 au laboratoire national de Los Alamos, aux États-Unis, Mecque de la physique de la complexité, pour un post-doc, avant un recrutement au CNRS deux ans plus tard. Au programme de son activité de recherche : physique du désordre, informatique théorique ou encore algorithmes d'acquisition comprimée. "Classiquement, lorsque l'on fait des photos ou pour une IRM médicale, on commence par acquérir les données avant de les compresser. Or la possibilité d'effectuer la compression au moment même de la mesure permettrait un gain de temps considérable ", précise Lenka Zdeborová.

Sur cette démarche, aux confluents de l'abstraction et d'applications on ne peut plus concrètes, elle explique : "J'aime les mathématiques car elles permettent des réponses sans incertitude. Pour autant, je me sens physicienne en ce sens que la formulation d'une conjecture pour laquelle on dispose d'arguments solides permet d'avancer sans forcément disposer de tous les détails d'une démonstration. "

Tout comme le permettent à ses yeux les interactions avec ses pairs. Meilleure preuve? L'investissement constant de Lenka Zdeborová dans l'organisation de conférences, de colloques ou de séminaires, dont l'un rassemble régulièrement les spécialistes d'Île-de-France de son domaine. "La recherche va au-delà des collaborations: c'est aussi des amitiés, d'où l'importance de parler, d'échanger, de se voir. " Bien au-delà de la question de savoir s'il s'agit de maths ou de physique !

Mathieu Grousson, journaliste

[1] www.cnrs.fr/fr/recherche/prix/medaillesbronze.htm

- Web

Site de I'IPhT : http://ipht.cea.fr/

Site de I'Institut de physique du CNRS :

www.cnrs.fr/inp/spip.php?article2617 